\title{
Matching human pathology is essential for validating OCT imaging to detect high-risk plaques
}

We thank Mark Brezinski for his Correspondence (Capabilities, limitations, and misconceptions of using OCT to assess vulnerable plaques. Nat. Rev. Cardiol. doi:10.1038/nrcardio.2014.62-c1 $)^{1}$ on our Review (Clinical classification of plaque morphology in coronary disease. Nat. Rev. Cardiol. 11, 379-389; 2014), ${ }^{2}$ and for his comments and clarifications. We agree that the phenomenon of light scattering, rather than the high resolution of the modality, might restrict the penetration depth of optical coherence tomography (OCT).

However, we would like to emphasize that there are no "glaring inconsistencies throughout the Review", ${ }^{1}$ and that the pathological differences between lipid pools and necrotic cores presented in our article are very carefully chosen terms; they are not identical nor used interchangeably. We have clearly stated that lipid pools are present in lesions with pathological intimal thickening, are located close to the medial wall, and consist of proteoglycans and lipid (where lipid can be identified as microvesicular droplets using oil red-O staining). ${ }^{2}$ Conversely, fibroatheromas are categorized as being 'early' or 'late'. Early fibroatheromas are characterized by macrophages infiltrating into the lipid pool with focal loss of proteoglycans and collagen type III, and contain free cholesterol. ${ }^{3}$ Late fibroatheromas have a necrotic core that is different from lipid pools, because they have discrete areas of cellular debris, no proteoglycans, and more numerous free cholesterol clefts. ${ }^{3}$ Differentiating between lesions of pathological intimal thickening and fibroatheromas is important because the latter progress to thin-cap fibroatheromas and possibly rupture. To the best of our knowledge, OCT cannot be used to distinguish between the two forms of lesion.

Necrotic cores can also embolize in the distal circulation after fibrous-cap rupture, leaving behind an easily identifiable cavity on OCT images. ${ }^{4}$ In a personal communication to J.N., Dr Brezinski claimed that an important difference in the definition of plaque rupture using histology versus OCT is the presence of an intraplaque cavity. $\mathrm{He}$ stated that, whereas researchers in imaging studies have applied a definition of plaque rupture as being a disrupted thin fibrous cap together with an intraplaque cavity, the mandatory presence of an intraplaque cavity has not been reported in histopathology studies. He also asserted that the highpressure contrast flush along with overlying thrombus obscuring the underlying plaque morphology might result in a false impression of an intraplaque cavity when imaging plaque rupture with OCT.

In our Review, we clearly show that foamy macrophages can be visualized as bright spots, and we report that these bright spots correlate with macrophages identified using immunohistochemical CD68 staining in coregistered sections. ${ }^{2}$ We disagree with the abstract by Phipps et al. that showed bright spots in multiple areas. ${ }^{5} \mathrm{We}$ have measured the peak intensity and also determined attenuation rate of various components of the intimal tissue, and show that the highest intensity is observed with foamy macrophages, followed by collagen seen in mature neointimal tissue rich in type I collagen after stenting. ${ }^{6}$ Platelet-rich thrombus gives a high-intensity image, but is far lower than that seen with foamy macrophages. ${ }^{6}$ Although yet to be published, we have observed that high-intensity images might also be seen with free cholesterol (that is, cholesterol clefts on histology). We are not aware whether the data from Phipps et al., presented in abstract form at two meetings, has yet been published as a full manuscript.

Evidence is available to support our statements that fibrous caps of ruptured plaques are substantially inflamed, and that two-thirds of coronary thrombotic events result from plaque rupture..$^{7-9}$ Burke et al. define acute plaque rupture as consisting "of a luminal platelet-fibrin thrombus continuous with an underlying lipid-rich core. The connection between the thrombus and the lipid core was through a disrupted thin fibrous cap infiltrated by macrophages." Kolodgie et al. state that "ruptured plaques showed extensive macrophage infiltration of the fibrous cap". ${ }^{8}$ Finally, Bentzon et al. report that "ruptured caps contain fewer smooth muscle cells and less collagen than intact caps, and smooth muscle cells are usually absent at the actual site of rupture. At the same time, infiltrating macrophages degrade the collagen-rich cap matrix." 9 Therefore, sufficient evidence exists that macrophages are present at sites of ruptured caps. In conclusion, we believe that OCT images would be best understood by matching them with the pathology of each lesion, which would authenticate the use of OCT imaging and establish the capabilities and limitations of this modality for detecting high-risk plaques.

Cardiovascular Pathology Inc., 19 Firstfield Road, Gaithersburg, MD 20878, USA (R.V., F.O., M.J.). San Giovanni Addolorata Hospital and CLI Foundation, Via Amba Aradam, 8, Rome 00184, Italy (F.P.). Icahn School of Medicine at Mount Sinai, One Gustave L. Levy Place, New York, NY 10029, USA (J.N.). Correspondence to: R.V. rvirmani@cvpath.org

Competing interests

The authors declare no competing interests.

1. Brezinski, M. E. Capabilities, limitations, and misconceptions of using OCT to assess vulnerable plaques. Nat. Rev. Cardiol. http:// dx.doi.org/10.1038/nrcardio.2014.62-c1.

2. Otsuka, F., Joner, M., Prati, F., Virmani, R. $\&$ Narula, J. Clinical classification of plaque morphology in coronary disease. Nat. Rev. Cardiol. 11, 379-389 (2014).

3. Kolodgie, F. D. et al. Intraplaque hemorrhage and progression of coronary atheroma. N. Engl. J. Med. 349, 2316-2325 (2003).

4. Di Vito, L., Prati, F., Arbustini, E., Crea, F. \& Maseri, A. A “stable" coronary plaque rupture 


\section{CORRESPONDENCE}

documented by repeated OCT studies. JACC Cardiovasc. Imaging 6, 835-836 (2013).

5. Phipps, J. E., Vela, D., Hoyt, T., Milner, T.

\& Feldman, M. Macrophages are not the only source of bright spots in intravascular optical coherence tomography images: a quantitative analysis of human atherosclerosis [abstract]. J. Am. Coll. Cardiol. 63 (Suppl.), A1754 (2014).
6. Nakano, M. et al. Ex vivo assessment of vascular response to coronary stents by optical frequency domain imaging. JACC Cardiovasc. Imaging 5, 71-82 (2012).

7. Burke, A. P. et al. Coronary risk factors and plaque morphology in men with coronary disease who died suddenly. N. Engl. J. Med. 336, 1276-1282 (1997).
8. Kolodgie, F. D. et al. Localization of apoptotic macrophages at the site of plaque rupture in sudden coronary death. Am. J. Pathol. 157, 1259-1268 (2000).

9. Bentzon, J. F., Otsuka, F., Virmani, R. \& Falk, E. Mechanism of plaque formation and rupture. Circ Res. 114, 1852-1866 (2014). 